\title{
Providing Core Curriculum Model for Second Grade of High School
}

\author{
Esmaeil Kazempour ${ }^{1}$, Hamze Abar $^{2}$ \\ ${ }^{1}$ Associate Professor, Department of Educational Sciences, Tonekabon branch, Islamic Azad University, \\ Tonekabon, Iran. \\ ${ }^{2}$ M.A Education planning, Tonekabon branch, Islamic Azad University, Tonekabon, Iran.
}

\begin{tabular}{l} 
ARTICLE INFO \\
\hline Keywords: \\
Curriculum, Core \\
Curriculum, Patterns of \\
Curriculum, Second high \\
school course
\end{tabular}

\begin{abstract}
This study is going to present a theoretical model for developing the core curriculum. The present study was conducted in analytical descriptive method. The statistical population of the study consisted of experts and experts in the study including 200 of the experts and experts in the literature. a questionnaire was set in 80 questions from a Likert scale, the reliability of the questionnaire was estimated at $93 / 99$. in order to evaluate the validity of the questionnaire, the questionnaire was used after the sampling adequacy (KMO) and the Bartlett test. to analyze the data from descriptive and inferential statistics, conceptualization process, factor analysis (principal component analysis) and path analysis were used. the results showed that the core curriculum is modeled in six factors. In the final step, using factor rotation, the simple structure of factors was obtained and factors under the titles of learning, content and materials, organization of learning opportunity, design strategies and program implementation, learning strategies, program execution quality were named and the conceptual model was designed according to these six factors. And the statistics of path analysis in the form of a model showed that each of the six factors had sufficient importance to the core curricula.
\end{abstract}

\section{Introduction}

In today's complex world, man needs comprehensive perspective to solve the problems ahead. In the meantime, there is only the interdisciplinary studies that cause the environment surrounding individuals, especially the academic environment, and the studies of experts, leading to the dynamics of the educational system and consequently the development and reform of culture. In addition, interference in content, methods of research, principles and theories have destroyed common frontiers of sciences.

The core (central) grade curriculum, one of the approaches to student - oriented curriculum, is a program at the core and center of the educational system, and is often organized by the Ministry of Education for all stakeholders, but colleges are at undergraduate levels for the curriculum. The

* Corresponding Author E-Mail Address: hamzeh.abar@yahoo.com 
core curriculum is the basis of learners training, and includes courses of writing, reading, foreign language, experimental and human sciences, and to become a contemplative individual not only on their education, but also in their life (education Saskatchewan, 2010).

In the dictionary, the word "core" is defined as the nucleus and center of something and the core curriculum (common grade) is defined as a set of courses that are considered as centers and for all learners or compulsory school systems. The original (core) curriculum is also a fundamental part of education, as it establishes the necessary skills or shared cultural knowledge (Yemeni, 1390, p. 1264).

This type of curriculum developed in the $1930 \mathrm{~s}$ and $1940 \mathrm{~s}$, ignoring various forms of students ' knowledge boundaries (Farmahini farahani, 1390).

The core (Central) curriculum can be regarded as the main themes, skills and information necessary for success in education and later stages of life. It draws our attention to a collection of knowledge and skills that are taught to all stakeholders. The origin and centrality of it makes primary and initial training not only as a certain stage in education, but an essential stage in inclusive life (Education Saskatchewan 2010).

There are many criticisms of the common base curriculum, including the most important of which is that the pattern has not been successful as it is claimed. The reason for this is that the implementation of the common base pattern involves the expertise and scientific ability of the teacher in many subjects, the extensive differences between teachers and detailed planning between teachers (Fathi and vajargah 2011).

The common grade curriculum is intended to provide a training program for all students to train basic skills and introduce a wide range of new training programs. It also includes processes and knowledge required to achieve wider goals as recognized by the training program and the Education Review Committee (Saskatchewan Education, 2013).

Yamani et al. (2011) achieved these results. It is necessary that the curriculum developers, using effective methods of physicians, provide the necessary and necessary training of the learners according to the needs of the community in order to provide the patients with the desired services, and the vision for the need for review. Do not lose curriculum and reduce the burden of curriculum content.

In another paper from Suschkun's (2007) the teachers can use it as a tool to integrate the components and plans of the class as a means of integrating components and core curricula for their class exercises. These results are required to provide students with effective approaches and feedback, in order to provide favorable services to patients, and not lose sight of the curricula of the curriculum and reduce the additional burden of the curriculum content.

In the JWG study (2009), one of the drawbacks and shortcomings of academic curriculum is the tendency to emphasize individual integrity, ignoring the relation between people as well as between the scientific experience of students and those beyond the school. Unfortunately, evidence suggests that high school students, who have completed more subjects than their predecessors, have increasingly weakened their view of the attractiveness of their academic work, their significance, and the likelihood of their usefulness in their lives later. This trend points out the necessity of providing opportunities for students in the core curriculum to relate their experiences between subjects and individuals and to relate their academic experiences to life outside the school. Thomas Hall's research (2010) examines the provision of a collaborative scientific experience for All undergraduate students have also been studying basic qualifications in writing, foreign language, scientific research, cultural knowledge, and interdisciplinary scholarship. It has been pointed out in this study. Schools may add other academic requirements, and students do not 
consider it when planning their time at the entrance to the school. -Meral (2010) pointed out in a paper that a core curriculum can be at levels Different, for example, global, national, and organizational. An analysis of policies and policies is an official field in the field of education and has led to the stimulation and deliberate motivation of discussing, analyzing, evaluating and advising on educational policies, as well as issues Political, legal, and tax related issues. The Whitica Research (2012) refers to a joint national syllabus curriculum as a means to activate and manage educational changes in the development of the Finnish school system. A national core curriculum includes the main objectives and content of training for all schools, as well as the phases and missions, values and training structure. It also describes the concept of learning and goals for the development of the learning environment, school culture and work methods.

In Brown's research (2012), a core curriculum for masters undergraduate, prepares students for professional excellence, civic responsibility, and service to society, especially from those who need the most. The common ground curriculum also emphasizes critical judgment, clear expression, ethical decision making, and attention to others. The common ground curriculum can play an important role in determining the needs and interests of the students.

Generally, a core curricula can play an important role in promoting and improving school learning and organizing it to active learning and important role in relating students ' learning to real life and social issues. In all of this research, what is the core curriculum and what is the core curriculum? But according to the above description, the present study tries to answer the fundamental question which is the core curriculum model which is appropriate for the secondary school? In order to achieve the goal, the following questions were discussed.

1. What are the components of the common basic curriculum for the second high school?

What elements should be considered for the second-level high school curriculum model?

3. To what extent is the designed model to be valid of curriculum expert's perspective?

4. To what extent is the designed model to be reliable from the perspective of curriculum specialists?

5. What is the appropriate model for combining the elements of the common foundation curriculum for secondary education?

6. Is the core curriculum model sufficiently high at the second grade of secondary education?

\section{Methodology}

The present study is a descriptive-analytic study. The statistical population of the present study includes Iranian experts and specialists in education. The sample consists of 200 people. The data gathering tool was a questionnaire of 80 questions from the Likert spectrum. The content validity of the questionnaire questions was reviewed and approved by the experts in the field of curriculum planning. Reliability of the questionnaire was calculated using Cronbach's alpha. And a questionnaire was conducted in a preliminary study on 25 people other than the sample population and the Cronbach's alpha was 0.93. Data analysis was done according to the nature of the research questions in two levels of descriptive and inferential statistics, conceptualization process, factor analysis, main factor analysis and path analysis.

\section{Findings}

The results of this study are presented in two parts of the descriptive and inferential findings related to the test of questions.

A) The description of the data: 
Table 1: Frequency distribution of respondents

\begin{tabular}{|l|c|c|}
\hline \multicolumn{1}{|c|}{ Position } & Frequency & Percentage \\
\hline Educational sciences specialist & 50 & 25 \\
\hline Educational sciences Expert & 150 & 75 \\
\hline Sum & 200 & 100 \\
\hline
\end{tabular}

\section{What are the components of the joint basic curriculum for the second secondary school?}

What are the components of the common basic curriculum for Secondary?

In this study, after studying the literature and the history of research, 80 components were extracted, based on which a questionnaire was developed that after the implementation of the statistical sample, factor analysis and effective factors and components were extracted and named. According to the results of 80 components and six The factors contributing to the creation of the second elementary high school textbook are the following: (the attention and emphasis on the needs and objectives of education, how to access learning and content sources and materials, determine and organize learning opportunities, learning methods, learning methods and learning methods, quality of the curriculum implementation, and consideration of the plan for achieving goals).

What elements should be considered for the proposed model of the common ground level curriculum in the second-high school level?

1. The amount of attention and emphasis on the needs and goals of education:

The elements involved in the new knowledge - development of self - development - the elimination of learning needs - the needs of emotional development, including elements that need to be considered in the needs of educational institutions.

2. How to access learning resources, content and materials:

Teacher's Ability to Use Educational Resources and Resources - Applying Visual Resources Paying attention to software and hardware- Ways of using the Web and using educational resources.

3. Determining and managing learning opportunities:

Development of creative activities for students - The need for flexibility in classroom space Linking meaningful learning to students the real loss is one of the things that need to be addressed in organizing learning opportunities.

4. Teaching methods-Learning and teaching methods:

Choosing teaching methods in the classroom and the psychological dimensions of teaching - the choice of teaching methods tailored to the educational space - the choice of teaching methods tailored to the educational space - the experience of teaching teachers - varied educational methods, and creating creative thinking among students.

5. The factors related to the quality of the curriculum implementation:

The control of pedagogical programs by the researchers - supervision, and oversight by monitoring institutions - the role of teachers in training courses - the role of teachers in training courses - the teacher 's role in teaching teachers - - how to implement the program of relevant regulatory factors. 6. Pay attention to the evaluation plan:

The use of student experiences in the assessment - paying attention to appropriate feedback from the outcome of the curriculum assessment in teaching learning process at the end - employs various strategies to evaluate the underlying factors of assessment. 
3 :What is the basis of the core curriculum model as well as the qualifications of the educational sciences?

In order to include all desired content, the initial questionnaire questions were reviewed by pundits in this field of subject planning and the match of the questions (elements) was examined with the subject and purpose of the research, and finally, on the basis of their views the necessary modifications were made.

B: Analysis of data (statistical tests)

4: To what extent is the pattern that is designed to be credible from the point of view of the specialist and planning curriculum?

In order to validate the model, a sample of 200 professionals and experts in education was selected. Based on the obtained data the analyzes required for each of the components of the commonly used (proposed) syllabus model are descriptive and inferential.

Table 2: Coefficient of reliability of core curriculum factors

\begin{tabular}{|l|c|c|c|}
\hline \multirow{2}{*}{ Factor } & \multicolumn{3}{c|}{ Indicator } \\
\cline { 2 - 4 } & Alpha & Components & Sample \\
\hline 1.learning sources, content and- materials & 0.867 & 18 & 200 \\
\hline 2.Quality of the curriculum implementation & 0.833 & 13 & 200 \\
\hline 3.Design strategies and optimal application -Implementation & 0.790 & 10 & 200 \\
\hline 4.Paying attention to educational needs and goals & 0.822 & 11 & 200 \\
\hline 5.organizing learning -opportunities & 0.811 & 8 & 200 \\
\hline 6.Ways of learning, teaching and training methods & 0.735 & 8 & 200 \\
\hline Total & 0.927 & 68 & 200 \\
\hline
\end{tabular}

The results of the above table show that the index of the core curriculum index has acceptable reliability $(927 /$.

To assess the adequacy of the model and whether this model is also applicable in the real environment, the index is used to answer the above question, factor analysis using principal components analysis.

The structural equation model is used to describe the following table.

Table 3: Assessment of the test K.M.O and Bartlett

\begin{tabular}{|c|c|c|}
\hline \multicolumn{2}{|c|}{ Kaiser-Meyer-Olkin Measure of Sampling Adequacy } & 0.654 \\
\hline \multirow{3}{*}{ Bartlett's Test of Sphericity } & Approx. Chi-Square & 10496.576 \\
\cline { 2 - 3 } & $\mathrm{df}$ & 2278 \\
\cline { 2 - 3 } & Sig. & 0.000 \\
\hline
\end{tabular}

The rate of K.M.O statistics in the table above shows that the factor analysis is acceptable at a moderate level and its results can be generalized to the statistical community. The calculated value for the Bartlett test at 001 /. Meaningful. Therefore, it can be concluded that the correlations between the items are suitable for factor analysis. It should be noted that 12 components with a high standard deviation were eliminated from the whole model. $(3,5,15,24,31,35,46,58,66$, $72,73,74$. 
Based on the findings, more subscriptions are less than 0.5 Which indicates the ability of the average factors in explaining the variance of the studied variables. According to the results, as variables as " Understanding the Student's Emotional Growth Needs "," The role of teachers' experience and expertise in reducing student anxiety stress "and" Attention to the development of individual development at the end of the evaluation "have the most common factor variance.

Table 5: Total variance of factors explained

\begin{tabular}{|c|c|c|c|}
\hline Value & Special value & Percentage of variance & Cumulative variance \\
\hline 1 & 12.929 & 10.015 & 10.015 \\
\hline 2 & 5.669 & 8.985 & 19.005 \\
\hline 3 & 4.131 & 7.463 & 26.463 \\
\hline 4 & 3.495 & 7.383 & 33.846 \\
\hline 5 & 3.226 & 7.158 & 41.005 \\
\hline 6 & 2.577 & 6.094 & 47.099 \\
\hline
\end{tabular}

The table above describes the description of the special values and the variance associated with each of the factors. As you can see, only six of the first factors are of prime importance. Therefore, it can be said that the elements of the curriculum can be reduced to six factors. According to the column of total squares of factor loads after the rotation, factors 1-6, respectively, were 10.01, 98.58, 463.7, 7383.7, 158.7, and $094.6 \%$ of the variance of the core curriculum variables, respectively Explain the core curriculum variables.and " This percentage is relatively acceptable in the factor analysis, and it can be ensured that the selected variables are appropriate for factor analysis."

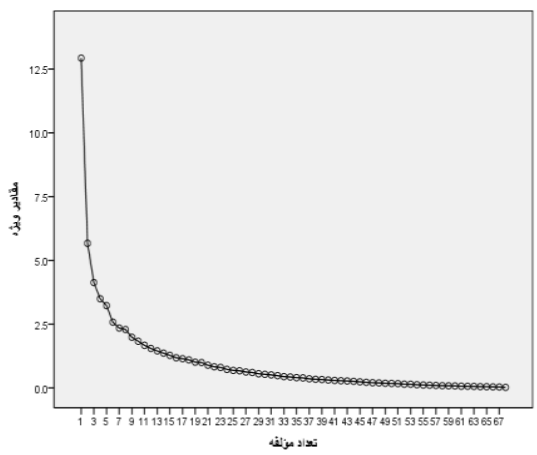

Chart 1 Specific values for Core curriculum variables

Based on the chart above, only six factors with special values are higher than 2 . Therefore, in the final analysis, these six factors have been used and other factors have been removed from the analysis.

Table 6: The coefficients of variables loaded in the first factor after the rotation

\begin{tabular}{|l|l|l|}
\hline Questions & Variable & \\
\hline 77 & Use of Teachers in the Evaluation of Educational Content Writing & 0.688 \\
\hline 78 & Considering the Growth of Students' Emotional Dimensions in Final Evaluation & 0.659 \\
\hline 6 & Matching the program with the perspectives and values of education & 0.558 \\
\hline 14 & Recognition of Equal Rights & 0.624 \\
\hline
\end{tabular}




\begin{tabular}{|l|l|l|}
\hline 50 & The role of teacher's experience and expertise in reducing anxiety & 0.621 \\
\hline 49 & The Necessity of Student-Centered Nuclear Program & 0.617 \\
\hline 23 & The Role of Using Materials and Educational Content in Lifelong Learning & 0.532 \\
\hline 43 & $\begin{array}{l}\text { The Role of Research Groups in the Development of the Intellectual Process and } \\
\text { Learning }\end{array}$ & 0.5 .6 \\
\hline 36 & Necessity to link meaningful learning with real life & 0.50 \\
\hline 39 & Necessity of Active Learning Positioning & 0.498 \\
\hline 57 & The Use of Participation in Learning Discussion & 0.497 \\
\hline 30 & Role of Educational Resources and Content in Motivating Students & 0.491 \\
\hline 21 & Necessity of written assignments in student learning & 0.47 \\
\hline 22 & The Role of Learning Resources and Content to Understanding Knowledge & 0.459 \\
\hline 17 & Need for teacher's ability to use educational resources & 0.428 \\
\hline 1 & The need to follow the development of a core curriculum & 0.426 \\
\hline
\end{tabular}

According to the above table, the most variables are consistent with theoretical foundations for learning resources, therefore, the first factor can be called "learning resources, content and syllabus".

Table 7: The coefficients of variables loaded in the second factor after the rotation

\begin{tabular}{|l|l|l|}
\hline Questions & Variable & Factor 2 \\
\hline 67 & regulatory barriers to adopting the plan & 0.69 \\
\hline 60 & Precise monitoring and control by regulatory authorities & 0.657 \\
\hline 64 & differences between program designed and implemented & 0.6 .6 \\
\hline 62 & $\begin{array}{l}\text { Necessity of the role of instructors in training courses on the implementation } \\
\text { of the program }\end{array}$ & 0.586 \\
\hline 13 & Considering the cultivation of elite people & 0.584 \\
\hline 18 & Incarnation of visual resources in the use of learning resources & 0.544 \\
\hline 70 & Use Student Experiences in Valuation & 0.522 \\
\hline 59 & Controlling educational programs and materials by researchers & 0.504 \\
\hline 75 & Suitability of exchange rate strategies with learning objectives & 0.493 \\
\hline 69 & $\begin{array}{l}\text { The fit of student-centered mental and physical fitness assessment } \\
\text { practices }\end{array}$ & 0.485 \\
\hline 69 & $\begin{array}{l}\text { Student Achievement for Goals proper feedback from the outcome of the curriculum } \\
\text { Considering the propess } \\
\text { evaluation process in the learning process }\end{array}$ & 0.425 \\
\hline 71 & The Impact of Educational Methods on Psychological Dimensions of Teaching & 0.418 \\
\hline 47 & \multicolumn{2}{|l}{} \\
\hline
\end{tabular}

According to the above table, based on theoretical foundations, the components of the second factor are more in relation to how the program is implemented. So the second factor can be called the "quality of curriculum implementation." 
Table 8: The coefficients of variables loaded in the third factor after the rotation

\begin{tabular}{|l|l|l|}
\hline Questions & Variable & Factor 3 \\
\hline 56 & $\begin{array}{l}\text { the Role of Mastery Learning in Reducing the Difference Between Success and } \\
\text { Ability }\end{array}$ & 0.648 \\
\hline 28 & The Role of Technology in Teacher-Student Interaction & 0.564 \\
\hline 33 & Role of parallelizing learning lessons & 0.553 \\
\hline 2 & tendency to research and produce new knowledge & 0.509 \\
\hline 11 & Attention to the diversity of the educational system & 0.507 \\
\hline 80 & Attention to evaluation strategies in learning experiences & 0.507 \\
\hline 61 & Necessity of the courses needed to implement the program & 0.485 \\
\hline 27 & Content fit with the student's mental and physical abilities & 0.452 \\
\hline 29 & Attention to hardware in the production of curriculum & 0.401 \\
\hline 79 & $\begin{array}{l}\text { Attention to the development of individual development at the end of the } \\
\text { evaluation }\end{array}$ & 0.364 \\
\hline
\end{tabular}

According to the components in the table above, most of the components are in the main strategies for design and implementation of the program. Therefore, the third factor can be called the "design and implementation strategies of the program".

Table 9 - The coefficients of variables loaded in the fourth factor after the rotation

\begin{tabular}{|l|l|l|}
\hline Questions & Variable & Factor 4 \\
\hline 19 & teacher autonomy in compiling educational content & 0.673 \\
\hline 10 & Roles to Improve Student's Perception of Life and Interaction with the World & 0.673 \\
\hline 8 & Understand the program of the student's emotional growth needs & 0.556 \\
\hline 20 & Program flexibility versus environmental conditions & 0.552 \\
\hline 12 & Attention to improve student understanding and character & 0.519 \\
\hline 4 & Proximity to educational goals & 0.511 \\
\hline 9 & Attention to gender equality in access to technology & 0.472 \\
\hline 54 & The Need for Justice in Teaching Methods Given the Individual Difference & 0.447 \\
\hline 68 & $\begin{array}{l}\text { The necessity of the terms of the program in terms of temporal and spatial } \\
\text { domains }\end{array}$ & 0.427 \\
\hline 45 & The role of educational methods in expanding the culture of learning conflict & 0.393 \\
\hline 7 & Attention to meet learning needs & 0.386 \\
\hline
\end{tabular}

According to the above table, the components of the fourth factor are more relevant to educational needs. Therefore, the fourth factor can be called "attention to educational needs and goals".

Table 10 - The coefficients of the variables loaded in the fifth factor after the rotation

\begin{tabular}{|l|l|l|}
\hline Questions & Variable & Factor 5 \\
\hline 38 & The need for active participation of students in learning & 0.687 \\
\hline 55 & Role of teaching methods in preparing learning opportunities & 0.661 \\
\hline 51 & Role of teaching methods in preparing learning opportunities & 0.661 \\
\hline 44 & The need for participatory planning by the teacher and the student & 0.661 \\
\hline
\end{tabular}




\begin{tabular}{|l|l|l|}
\hline 26 & The need for Understanding the Use of Resources and Educational Content & 0.516 \\
\hline 52 & The need for a comfortable communication between learners and teachers & 0.488 \\
\hline 41 & Teacher's technical ability to organize learning & 0.430 \\
\hline 40 & The need for content selection by the teacher and student in the learning process & 0.337 \\
\hline
\end{tabular}

Based on theoretical foundations, components of the fifth factor are more about the organization of learning status. This factor can be named "organizing learning opportunities".

Table 11 - Load variables coefficients in the sixth factor after the rotation

\begin{tabular}{|l|l|l|}
\hline Questions & Variable & Factor 6 \\
\hline 34 & The Need for Developing Creative Activities for Students & 0.689 \\
\hline 32 & Paying attention to professional ethics & 0.689 \\
\hline 63 & The Need for Teacher Teaching Quality & 0.669 \\
\hline 48 & Matching the choice of teaching methods with time and learning environment & 0.493 \\
\hline 53 & Considering various conditions and signs & 0.49 \\
\hline 25 & roles in software production & 0.433 \\
\hline 42 & $\begin{array}{l}\text { The Effect of Understanding and recognition in Learning Methods to Reducing } \\
\text { Students' Anxiety }\end{array}$ & 0.430 \\
\hline 76 & Attention to fair resources and training materials in final evaluation & 0.407 \\
\hline
\end{tabular}

According to theoretical foundations, the most important components of the sixth factor are the need to adopt different learning methods. Therefore, this factor can be called "learning methods and teaching methods".

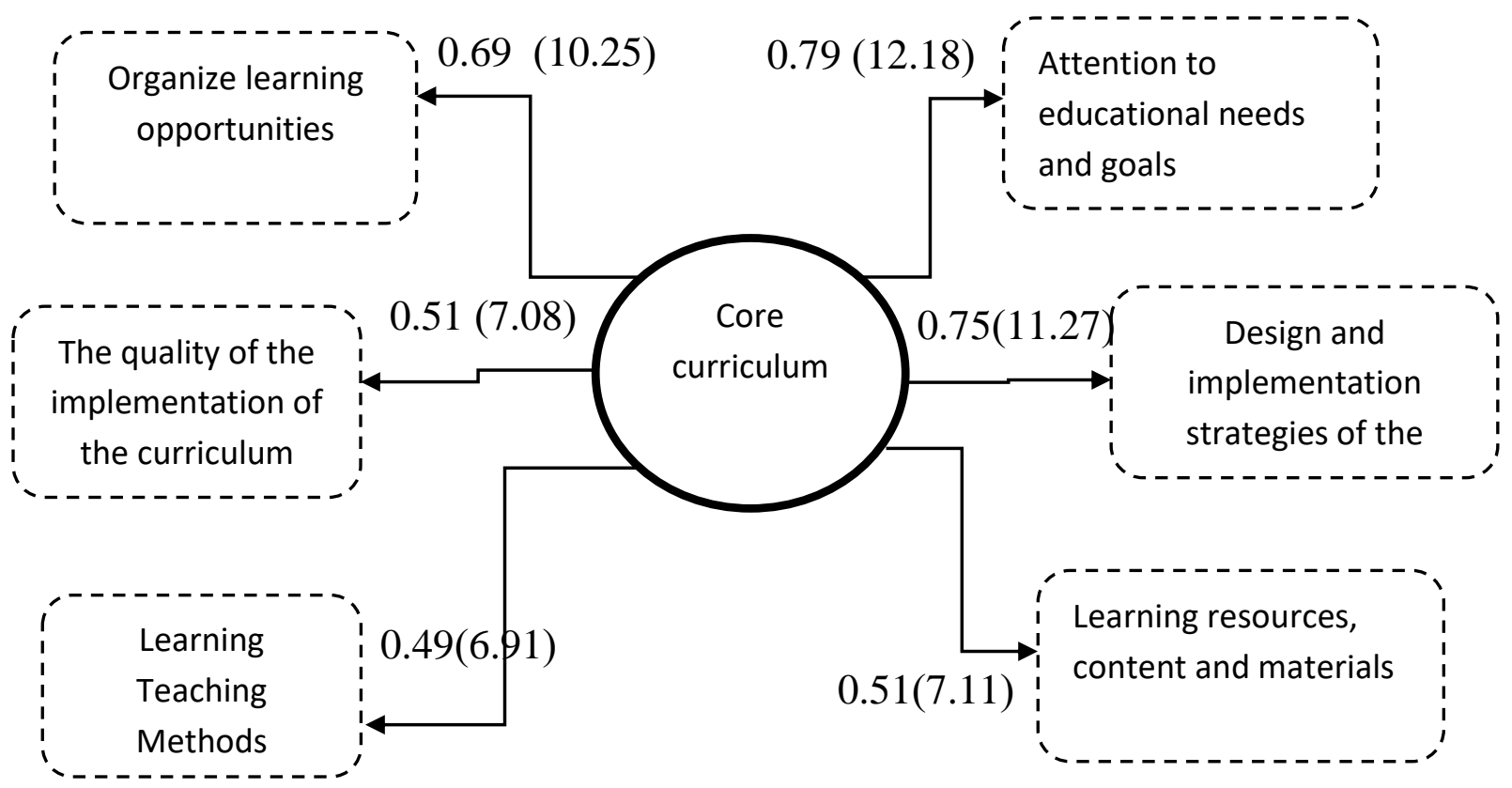

Proposed Final Pattern of Core Curriculumm

$$
\text { Chi-squar }=19.55 \quad \text { DF }=9 \quad \text { P-valu }=0.02087 \quad \text { RMSEA }=0.077
$$

Figure 2: Structural equation for the relationship between the core curriculum and the main components of the program 
The above diagram shows the relationships between the core curriculum and its main components. As you can see, all relationships are from the middle to the top level and with respect to the values of $t$, all relations are meaningful. Based on the RMSEA index which is close to 0.05. Can be said the final model has a good fit and can be generalized to the real environment. As seen in Chart (2) the data contained in the hidden variable arrow (curriculum) to the agents is considered as factor load. The most load factor is 0.79 it is related to the "attention to educational needs and goals" factor., It is calculated for factor load factor about "learning resources, content and materials" 0.51, for the "organizing learning opportunity" factor 0.69, For "design and implementation strategies" 0.69 , For the "Learning Teaching Methods" factor 0.49, And for "the quality of the program" 0.51. The values of the parentheses have a significant level of factor load observed at the level of 0.05. All values are higher than 1.96 ( $\mathrm{t}$ value is 95\%). This represents the meaning of all coefficients 6 - Is the Common Elementary School Principle Model Sufficient?

Table 12 - The adequacy of the curriculum design model

\begin{tabular}{|l|l|l|l|}
\hline Factor & \multicolumn{3}{|c|}{ Index } \\
\hline Name & NFI & GFI & AGFI \\
\hline 1 learning resources, content and syllabus & 0.83 & 0.89 & 0.78 \\
\hline 2 quality curriculum implementation & 0.82 & 0.84 & 0.77 \\
\hline 3 Strategic design and implementation strategies & 0.82 & 0.87 & 0.77 \\
\hline 4 Attention to educational needs and goals & 0.85 & 0.88 & 0.77 \\
\hline 5 Organize learning opportunities & 0.82 & 0.86 & 0.77 \\
\hline 6 Learning Teaching Practices and Educational Methods & 0.80 & 0.92 & 0.77 \\
\hline
\end{tabular}

At first, several indicators have been considered for the proposed model's suitability. One of these indicators is the Fit Fitness Index (GFI). If the greater calculated value is closer to a range of the index being between zero and one, represents a better fit of the model. Another indicator of goodness of fit is adjusted (AGFI).The value (AGFI) is from zero to one, and the values close to one is a good sign of fit the model. In the six models presented, all values are close to the Criterion. Therefore, it can be said that the model has a fair fit. It is also possible to calculate the index (NFI), which is close to 0.9 that Indicates fitness is acceptable. Thus, the proposed model has an acceptable fit with the predetermined theoretical model and can be generalized to the real environment.

Table 13- Pearson Correlation Matrix between the main components of the common basic curriculum

\begin{tabular}{|l|c|c|c|c|c|c|}
\hline \multicolumn{1}{|c|}{ Component } & 1 & 2 & 3 & 4 & 5 & 6 \\
\hline 1 learning resources & 1 & $0.235^{* *}$ & $0.453^{*}$ & $0.352^{* *}$ & 0.366 & $0.271^{* *}$ \\
\hline 2 Quality run & $0.235^{* *}$ & 1 & $0.292^{*}$ & $0.410^{* *}$ & $0.465^{* *}$ & $0.272^{* *}$ \\
\hline 3 Design and implementation strategies & $0.453^{* *}$ & $0.292^{*}$ & 1 & $0.617^{* *}$ & $0.498^{* *}$ & $0.346^{* *}$ \\
\hline 4 Attention to needs & $0.352^{* *}$ & $0.410^{* *}$ & $0.617^{* *}$ & 1 & $0.531^{* *}$ & $0.413^{* *}$ \\
\hline 5Organize learning & $0.366^{* *}$ & $0.465^{* *}$ & $0.346^{* *}$ & $0.531^{* *}$ & 1 & $0.319^{* *}$ \\
\hline 6 Learning Methods & $0.271^{* *}$ & $0.272^{* *}$ & $0.346^{* *}$ & $0.413^{*}$ & $0.319^{* *}$ & 1 \\
\hline
\end{tabular}

** Significant level at level $0.01 \quad *$ Significant level at level 0.05

The table above shows the correlation coefficients between the main components of the curriculum. The results indicate that there is solidarity among all factors at 0.001 . There is the 
highest correlation between the component of "attention to needs" and "the organization of learning to the extent" (0.531) and the lowest correlation is calculated between "learning resources" and "performance quality".

\section{Discussion and Conclusion}

Based on factor analysis by main component analysis, the questionnaire was saturated with six factors and then, the factors related to each factor were determined with its factor load factor. Finally, based on the identified factors, the proposed model of the core curriculum was presented. In that level, the impact of each factor on the core curriculum was calculated. In Suschkwan's research (2007), Emphasis on materials and rules for the education of students which is in line with the proposed model strategy. Brown's Curriculum Model (2012) emphasizes critical judgment, clear statement, ethical decision-making and attention to others. Considering the factor of attention to needs in the present model, a kind of synergy between the two patterns is observed. In the Whitica Pattern (2012), emphasis is placed on the Framework of National Core Curriculum Includes the main goals and main content of education for all school people, as well as stages and mission and values. It also describes the concept of learning and goals for developing a learning environment, school culture, and work methods. Finally, it can be deduced that in the present era, the concept of a curriculum is broader than the presentation of curriculum vitae, this is to anticipate all activities that a student under the leadership of a teacher teaches at school (Somewhere outside it) to achieve specific goals you must do. In other words, the curriculum includes all the experiences and learning opportunities officially and informally under the supervision and responsibility of the educational system, in order to create a favorable change in the knowledge, skills and attitudes of learners and its performance and effectiveness are evaluated. This conclusion can be drawn in the present model with respect to the determined factors, that curriculum design requires attention to many components So that the curriculum is designed to be a guide to good practice in the education process.

In the expert's view, the curriculum consists of several elements. The important thing is that each of the elements and elements also have a different place in the curriculum. In the present model, "learning resources, content and materials" was one of the factors that has more components. This suggests the need for basic preparations for implementing a curriculum. In other words, the prerequisite for the implementation of the curriculum is the preparation of resources and learning content which can play a role in the quality of the program. The interrelationships between the curriculum factors can be deduced that actual improvement of the curriculum requires making the appropriate changes in the set of these factors.

\section{References}

Brown (2012) Drahmann Academic Advising and Learning Resources Center Santa Clara University Core Curriculum Guid.

Erja VI tikka (2012) THE FINNISH NATIONAL CORE CURRICULUM: STRUCTURE AND DEVELOPMENT.

Fathi vagarghah, Kourosh (2011) Principles of Curriculum Planning. Tehran Iran Publications, Seventh Edition.

Formahini Farahani, Mohsen (2011) Descriptive Culture of Educational Sciences. Tehran Shabahang Publication.

Saskatchewan Ministry of Education. (2007) Principles, Time Allocations, and Credit Polic. Regina, SK: Government of Saskatchewan 
Saskatchewan Ministry of Education. (2010). Core Curriculum: career Education. Regina, SK: Government of Saskatchewan

Saskatchewan Ministry of Education. (2010). Core Curriculum: English Language Art. Regina, SK: Government of Saskatchewan

Saskatchewan Ministry of Education. (2010). Core Curriculum: English Language Art. Regina, SK: Government of Saskatchewan

Saskatchewan Ministry of Education. (2011). Core Curriculum: art education. Regina, SK: Government of Saskatchewan

Stanley Thomas Hall (2011). Undergraduate Core Curriculum.

Talebzadeh, Nobarian, Mohsen, Fathivagarghah, Kourosh (2003), Specialized Planning Curriculum, Printing: First, Tehran: Aeege.

Yamani, Niko.Firozabadi Nasrin. (2011). The main curriculum in medical education: the introduction of several approaches. Iranian Journal of Medical Education,11(9). 